\title{
Uso de imagens MODIS no monitoramento do fluxo de sedimentos no reservatório de Três Marias
}

\author{
Carlos E. T. Pinto ${ }^{1}$, Paulo H. B. J. Menezes', Jean-Michel Martinez ${ }^{3}$, Henrique L. Roig $\&$ Raúl A. E. Villar ${ }^{5}$ \\ ${ }^{1}$ Instituto de Geociências/UnB. Brasília, DF. E-mail: carlos.toniazzo@gmail.com (Autor correspondente) \\ ${ }^{2}$ Instituto de Geociências/UnB. Brasília, DF. E-mail: phjunker@hotmail.com \\ ${ }^{3}$ Institut de Recherche pour le Développement/Université Paul Sabatier, Instituto de Geociências/UnB. Brasília, DF. E-mail: jeanmichel.martinez@ird.fr \\ ${ }^{4}$ Instituto de Geociências/UnB. Brasília, DF. E-mail: roig@unb.br \\ ${ }^{5}$ Instituto de Geociências/UnB. Brasília, DF. E-mail: raulev@unb.br
}

\section{Palavras-chave:}

sensoriamento remoto

assoreamento

cor das águas

\section{R E S U M O}

Objetivou-se, neste trabalho, monitorar o fluxo de entrada de sedimentos no braço do Rio Indaiá no reservatório de Três Marias, MG, com base em dados das imagens de satélites. Foram adquiridas séries temporais de 12 anos das imagens MODIS (Terra e Aqua), compostas de 8 dias. Estimativas de reflectância da superfície foram calculadas usando-se o algoritmo MOD3R. Dados hidrossedimentométricos da estação de monitoramento do Rio Indaiá foram integrados às análises para se entender a relação entre as respostas das imagens de satélites e as medições de campo. Os resultados apontaram uma forte relação entre os dados de reflectância e a descarga sólida calculada no Rio Indaiá; há, também, uma forte variação da reflectância ao longo do braço do reservatório no sentido da montante à jusante, com altos valores de reflectância à montante e valores baixos à jusante. O percentual de registros válidos estimados nas imagens MODIS atendeu aos requisitos de monitoramento do fluxo de sedimentos enquanto as estimativas de reflectância da água mostraram grande potencial em relacionar as respostas espectrais das imagens de satélites com a variação da descarga sólida de sedimento no braço do Rio Indaiá.

\section{Key words:}

remote sensing

siltation

water color

\section{Use of MODIS images to monitor the sediment inflow into the Três Marias reservoir}

\begin{abstract}
A B S T R A C T
This study presents the monitoring of the sediment discharge from the Indaiá River into the Três Marias reservoir, based on the use of satellite images. A 12-year time series of MODIS 8-day image composites (Terra and Aqua onboard sensors) was used in this study. Estimates of the water surface reflectance were calculated using the algorithm MOD3R. River suspended sediment discharge records made available from the CEMIG hydroelectric company were integrated in the analysis to understand the variability of the remote sensing reflectance. The results showed a strong relationship between the reflectance data and the Indaiá river solid discharge. A strong variation of the reflectance is assessed within the reservoir (upstream towards downstream), with high reflectance values upstream (near the river mouth) and low reflectance values downstream. The estimated percentage of valid records in MODIS images met the requirements for monitoring the flow of sediment. Estimates of water reflectance show great potential in relating the sedimentation processes within the Reservoir as a function of time.
\end{abstract}

\section{INTRODUÇÃO}

Os reservatórios hidrelétricos são corpos de água importantes não só para a produção de energia elétrica mas também para o uso compartilhado ou usos múltiplos atendendo a outras necessidades básicas da população, como abastecimento, recreação e lazer, pesca comercial e controle de inundações, entre outros (ANEEL, 2000). A degradação desses sistemas aquáticos e da qualidade da água pode causar uma série de impactos ambientais entre eles o assoreamento e a perda da capacidade de geração de energia elétrica (Chanson, 1998). A qualidade da água pode ser degradada pela presença de nutrientes, microrganismos (Liu et al., 2003) e sedimentos em suspensão, também passíveis de carrear pesticidas e metais pesados adsorvidos causando, com isto, diversos problemas (Bradl, 2004).

Para Tonello (2005), os reservatórios de água constituem um recurso peculiar não somente pela sua amplitude de utilização, mas também por ser um excelente indicador ambiental da qualidade da manipulação do solo pelo homem, visto que atua como agente passivo frente aos acontecimentos que ocorrem à montante. Em síntese, as águas dos cursos que drenam uma região apresentam características físico-químicas próprias que refletem as atividades de uso da terra na respectiva bacia hidrográfica; este evento tem levado a uma demanda crescente por monitoramento da qualidade das águas nos rios 
e lagos buscando entender os impactos causados pelo uso das bacias hidrográficas. Hooper (1997) afirma que a eficácia do monitoramento hidrológico é função do número de parâmetros, da frequência e da distribuição espacial das medições na bacia. GEMS (2003) estimou, em um estudo global, que três quartos do planeta não têm um monitoramento da qualidade das águas adequado e isto não será alcançado em um futuro próximo.

No Brasil esta situação não é muito diferente, estudos de monitoramento e gerenciamento dos recursos hídricos ainda passam por muitas dificuldades, tanto em termos de análises quantitativas quanto qualitativas. A falta de dados é um dos principais problemas devido à dificuldade de instalação de instrumentos in situ e ao alto custo de manutenção e dos levantamentos de campo (Carvalho, 2008). A dimensão das bacias hidrográficas eleva os custos e dificulta análises periódicas com frequência espaço-temporal adequada; portanto, as técnicas de monitoramento convencionais das águas não conseguem acompanhar a crescente demanda de controle ambiental sendo necessário criar soluções alternativas.

Neste contexto, alguns dos parâmetros importantes para o entendimento da dinâmica dos reservatórios e da qualidade das águas podem ser estudados por sensoriamento remoto, como matéria orgânica dissolvida (Mannino et al., 2008; Zhu et al., 2011), sedimentos em suspensão (Kirk 1981; Mertes et al., 1993; Martinez et al., 2009; Wang \& Lu, 2010) e pigmentos das algas (Simis et al., 2005; Duan et al., 2007; Onderka \& Rodný, 2010). Assim, a aplicação de técnicas de sensoriamento remoto pode auxiliar na superação da limitação espaço-temporal dos métodos tradicionais in situ uma vez que adquire informações necessárias em diferentes escalas espaciais e temporais permitindo a análise estrutural de ecossistemas aquáticos.

No Brasil, trabalhos utilizando esta técnica aplicada a corpos de água continentais têm sido desenvolvidos demonstrando grande potencial para mapear a composição das águas (Novo et al., 1989; Rudorff et al., 2007; Ferreira \& Pereira Filho, 2009). Contudo, esses trabalhos não foram correlacionados às redes de monitoramento hidrológico buscando sua complementação e, na maioria dos casos, são estudos pontuais.

Recentemente, estudos usando sensores espaciais de alta taxa de revisita e resolução espacial moderada, como o Moderate Resolution Image Spectroradiometer (MODIS) têm sido utilizados para completar a rede de monitoramento e para avaliar a concentração de sedimentos em suspensão de superfície (SSS) nos rios da Bacia Amazônica (Martinez et al., 2009; Kilham \& Roberts, 2011; Villar et al., 2012). Esses trabalhos abriram novas perspectivas no monitoramento da qualidade das águas superficiais e a partir de dados dos sensores MODIS as imagens diárias fornecidas permitem realizar estimativas de refletância de qualquer ponto da superfície terrestre. Apesar da existência de outros estudos utilizando o sensor MODIS no Brasil (Valério et al., 2011; Nascimento et al., 2011), esses se restringiram a uma análise da correlação entre as informações espectrais e os parâmetros in situ sem incorporar a variável temporal nos estudos.

Tomando-se como base o exposto o objetivo deste trabalho foi monitorar o fluxo de entrada de sedimentos no braço do
Rio Indaiá no reservatório de Três Marias, MG, com base em dados das imagens de satélites. Pretende-se investigar o uso de imagens de satélite para monitorar parâmetros de qualidade da água, em particular os sedimentos em suspensão.

\section{MATERIAL e Métodos}

O reservatório de Três Marias localiza-se na região do alto São Francisco, no estado de Minas Gerais, entre os paralelos 18 e $21^{\circ}$ Sul e os meridianos $43^{\circ} 30^{\prime}$ e $46^{\circ} 40^{\prime}$ Oeste. A área drenada se estende desde as cabeceiras na Serra da Canastra, no município de São Roque de Minas, até a cidade de Pirapora, MG, abrangendo as sub-bacias dos rios das Velhas, Pará e Indaiá, além das sub-bacias dos rios Abaeté e Jequitibá.

De acordo com a classificação de Köppen, na região do Alto São Francisco ocorrem duas zonas climáticas: Cwa e Aw; A zona Cwa é caracterizada por um clima temperado brando, com verão quente (temperatura média do mês mais quente superior a $22^{\circ} \mathrm{C}$ ) e inverno brando (temperatura média do mês mais frio inferior a $18^{\circ} \mathrm{C}$ ) em que a estação seca coincide com o inverno; já a zona Aw possui clima tropical chuvoso, quente e úmido, é marcada por inverno seco e verão chuvoso; enfim, a temperatura média do mês mais frio é sempre superior a $18^{\circ} \mathrm{C}$.

O regime das chuvas é caracterizado por altas taxas pluviométricas, entre 1.150 e $1.450 \mathrm{~mm}$ anuais, com a ocorrência dos valores mensais máximos no período do verão e dos mínimos no inverno. Os meses de novembro a janeiro correspondem aos meses mais chuvosos com 55 a $60 \%$ do total anual precipitado. Os meses de junho a agosto são os mais secos com menos de 5\% de precipitação anual (Macedo, 2009).

Os principais afluentes que contribuem diretamente para o reservatório são: na margem esquerda os rios Borrachudo e Indaiá e na margem direita os rios Pará, Paraopeba e São Francisco (curso principal).

Este estudo foi focado no encontro do Rio Indaiá com o reservatório, denominado braço do Rio Indaiá (Figura 1). O Rio Indaiá nasce no município de Córrego Dantas, MG, próximo à comunidade de Cachoeirinha a cerca de $1.170 \mathrm{~m}$ de atitude; em seu percurso, de $226 \mathrm{~km}$, sua bacia hidrográfica é da ordem de $3.340 \mathrm{~km}^{2}$ indo desaguar no reservatório de Três Marias.

Para compreensão da variabilidade do ciclo hidrossedimentológico foram utilizados, neste estudo, dados de descarga sólida (sedimentos em suspensão e vazão total) de junho de 1999 a dezembro de 2002 da estação de monitoramento do Rio Indaiá localizada a $10 \mathrm{~km}$ do encontro do rio com o reservatório próximo à cidade Biquinhas, MG. Os dados foram disponibilizados pela CEMIG, empresa que opera e monitora o reservatório de Três Marias.

As imagens utilizadas são do sensor MODIS, lançado a bordo dos satélites Terra e Aqua. As imagens do sensor MODIS utilizadas, referem-se aos produtos de reflectância da superfície MOD09 (satélite Terra) e MYD09 (satélite Aqua), que são fornecidas previamente calibradas, georreferenciadas e corrigidas dos efeitos atmosféricos. No trabalho, são utilizadas imagens de dois tipos A1 e Q1, com resolução espacial de 500 e $250 \mathrm{~m}$, respectivamente. 


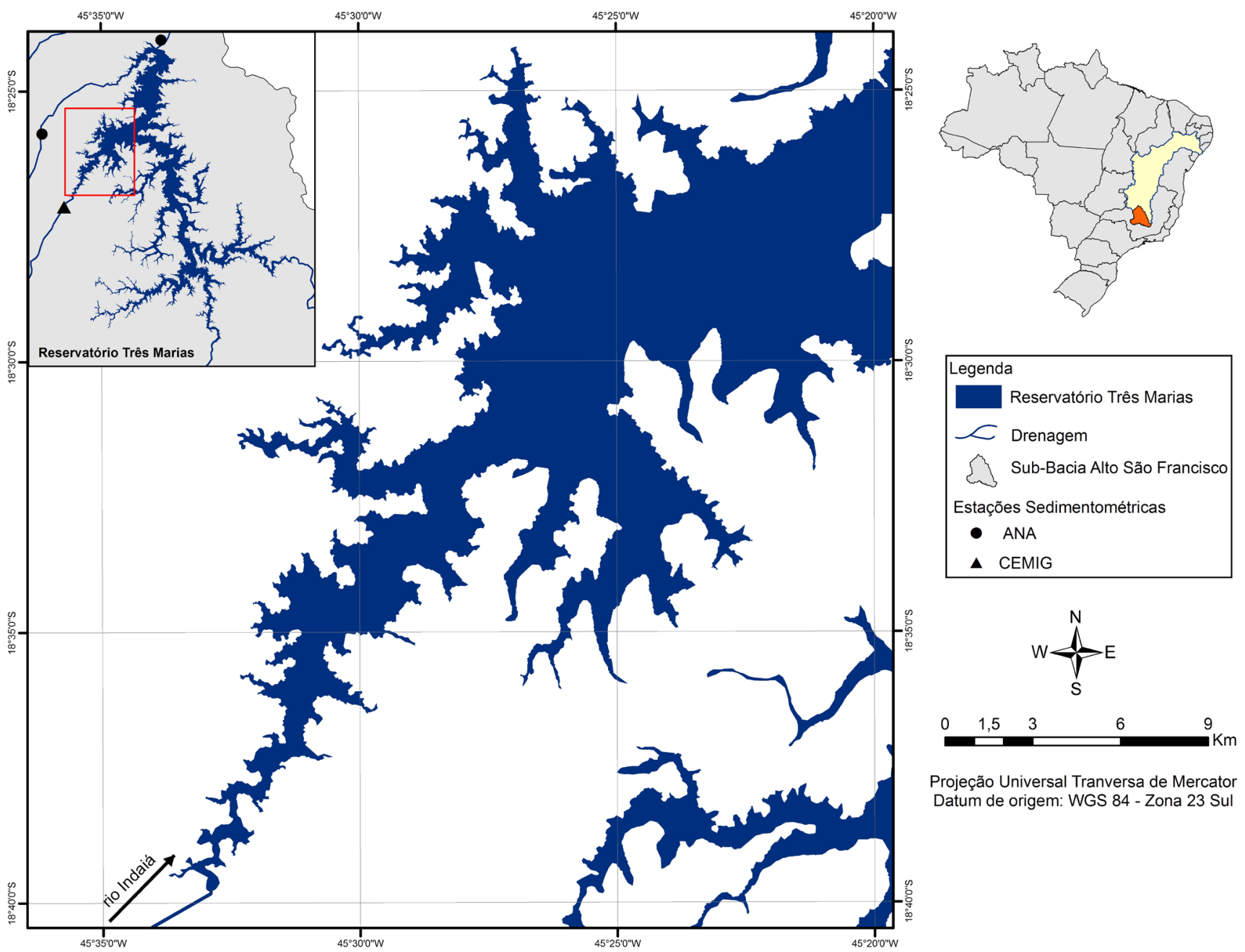

Figura 1. Braço do Rio Indaiá, reservatório Três Marias, MG

As imagens do tipo A1 são utilizadas apenas para obtenção das informações sobre a qualidade de processamento (pixel a pixel) enquanto as imagens Q1 são utilizadas para o processamento das duas bandas radiométricas que cobrem a região do vermelho (620-670 nm) e infravermelho (841-876 $\mathrm{nm}$ ). O sensor MODIS tem tempo quase diário de revisita sobre as áreas tropicais. Neste trabalho, tal como Martinez et al. (2009) e Villar et al. (2012), optou-se pelas imagens com composição de 8 dias devido à: i) redução do número de imagens com nuvens e, consequentemente, à melhoria da quantidade de dados a serem processados e ii) redução significativa do efeito da direção da refletância BRDF (reflectância bidirecional) e da atmosfera. Os produtos MODIS foram adquiridos a partir da base específica de dados GETMODIS (IRD) de fevereiro de 2000 até junho de 2012 representando, no total, 1024 imagens. A Figura 2 mostra as imagens selecionadas da área de estudo; a região escura no centro da imagem é referente ao reservatório, as regiões avermelhadas representam a vegetação (cerrado, mata ciliar e reflorestamento) e as regiões claras, solo exposto.

Em virtude da baixa resolução espacial do MODIS, a extração das medidas de reflectância da água é uma tarefa difícil e, dependendo da largura do rio ou reservatório e da geometria de aquisição da imagem, podem existir poucos pixels puros de água devido à mistura espectral com outros componente do
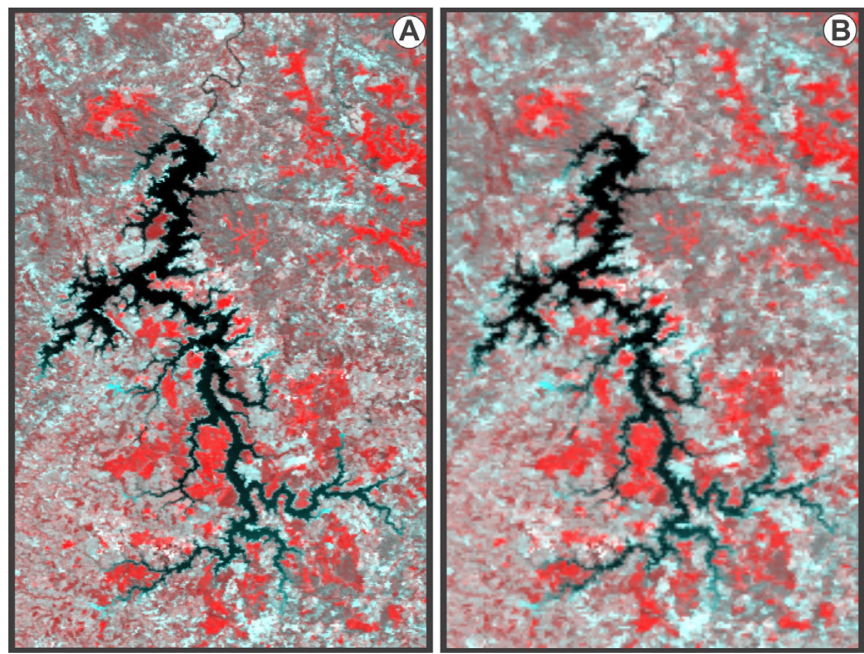

Figura 2. Imagens do sensor MODIS referentes ao produto de reflectância da superfície do satélite Terra. (A) Imagem MODIS produto Q1 com resolução espacial de $250 \mathrm{~m}$ e (B) Imagem MODIS do produto A1 com resolução espacial de $500 \mathrm{~m}$

sistema como por exemplo a vegetação da borda. Tal processo se torna árduo e praticamente impossível de ser executado manualmente quando se está trabalhando com séries temporais 
grandes, como no caso deste trabalho, no qual foram analisadas mais de 1000 imagens. A automatização deste processo é vital para minimizar o tempo, diminuir o erro operacional e garantir o mesmo processamento para todas as imagens. Para isto foi utilizado o algoritmo MOD3R (MODIS River Reflectance Retrieval) desenvolvido em linguagem JAVA pelo Institut de Recherve pour le Développement (IRD) (Martinez et al., 2009; Villar et al., 2012), que permite obter a reflectância dos endmembers de água em cada imagem; assim, o algoritmo desenvolvido determina com precisão e consistência os pixels de água pura na imagem, ao longo do tempo, independentemente dos tipos de morfologia dos rios ou reservatórios. Com a extração dos valores de reflectância das bandas do vermelho e do infravermelho das imagens MODIS, é possível determinar as concentrações dos sedimentos em suspensão e fitoplâncton presentes na água.

O programa apresenta uma interface simples para seleção da série temporal de imagens e escolha dos parâmetros necessários para os cálculos; são gerados, então, arquivos de saída com resumo das imagens processadas; daí, o resultado para cada imagem é acompanhado por um indicador de qualidade codificado entre um e três.

Para obtenção das estimativas de fluxo de sedimentos ao longo do braço do Indaiá, foram criadas máscaras nas áreas de interesse. Como não existe uma metodologia específica para essas análises, foram primeiramente determinadas regiões que recobriam todo braço, porém, os resultados dos testes apresentaram muitas variações, sendo necessário subdividir e estimar regiões menores e homogêneas. A Figura 3 mostra as máscaras criadas.

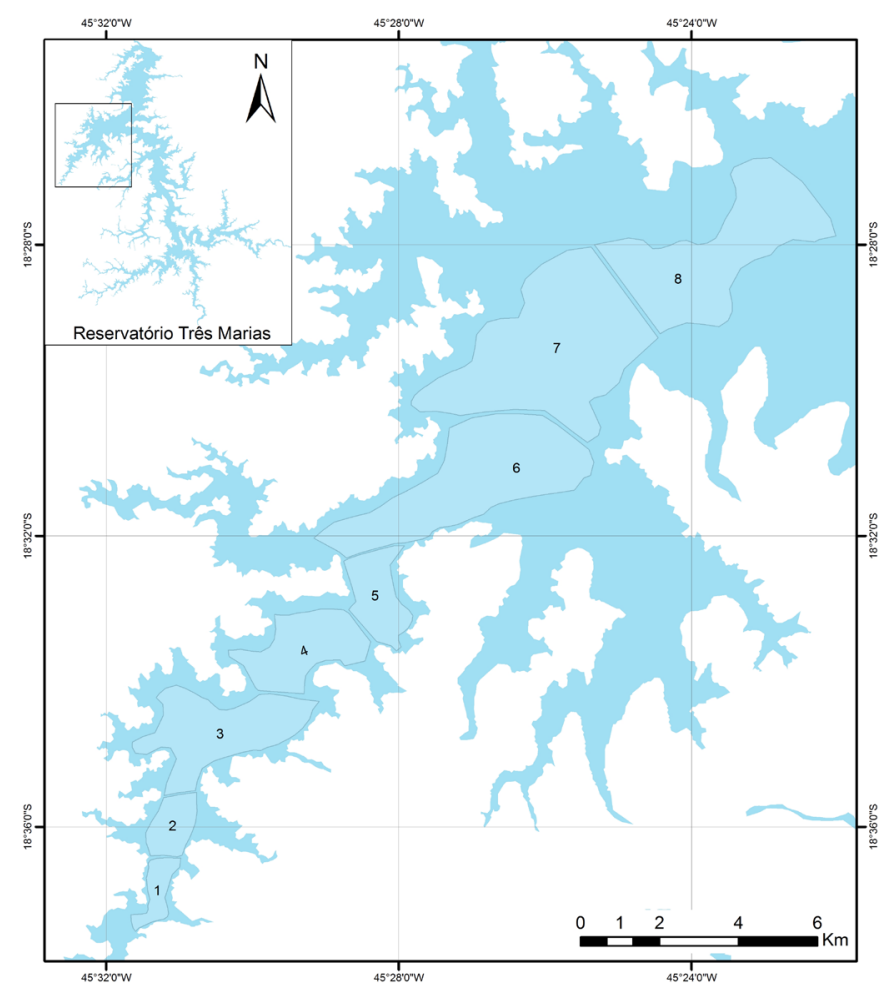

Figura 3. Máscaras criadas para análise da reflectância da água no braço do Rio Indaiá no reservatório Três Marias, MG
As etapas para a extração da reflectância do endmember da água usando o algoritmo MOD3R, segundo Martinez et al. (2009) são: 1) Os dados de entrada são as séries de imagens da área de interesse e a máscara da região que será estimada; 2) O primeiro passo é a extração dos pixels da máscara excluindo os pixels de má qualidade (coberto por nuvens) usando as informações fornecidas nas imagens de $500 \mathrm{~m}$; 3) Os valores de reflectância dos pixels são estimados e classificados em grupos homogêneos (clusters), através da metodologia estatística de K-means; 4) O programa estima o endmember de vegetação de maneira automatizada (cluster com maior diferença positiva entre o banda do vermelho e do infravermelho); 5) Cada cluster é testado como o potencial endmember de água e o grupo que produz o menor erro residual é selecionado solucionando o modelo linear de mistura espectral.

Este procedimento é centrado na determinação automática do endmember de água por testes sucessivos de um grupo de pixels em cada modelo linear de mistura, com vista a se encontrar o grupo que melhor descreve outros grupos de pixels misturados na água do rio.

Os valores de estimativas da reflectância na água são classificados por alguns critérios de qualidade, como ângulo de incidência solar, ângulo de visada do satélite, presença de aerossois e presença ou ausência de nuvens (banda de qualidade A1 MODIS). Para cada máscara criada uma tabela é registrada com valores de reflectância nas bandas do vermelho e infravermelho. Os valores das imagens de ambos os satélites (Terra e Aqua) foram fusionados, selecionando-se as de melhor qualidade, com intuito de obter uma série temporal com o maior número possíveis de dados.

\section{Resultados e Discussão}

A primeira abordagem do estudo hidrossedimentológico é entender a variabilidade sazonal do comportamento da vazão sólida diária no Rio Indaiá; após a análise da consistência dos dados foi traçado um gráfico que relaciona valores de descarga sólida diária da estação de monitoramento operada pela CEMIG no Rio Indaiá (Figura 4). Os dados de descarga sólida apresentam variação definida, com altos valores de descarga nos meses de novembro a janeiro, máximas de $321.000\left(\mathrm{t} \mathrm{d}^{-1}\right) \mathrm{e}$

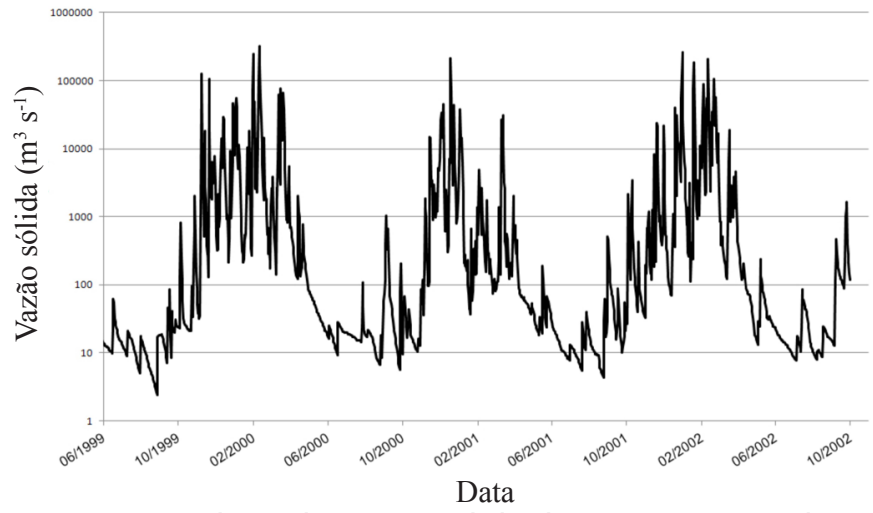

Figura 4. Fluxo de vazão sólida diária na estação do Rio Indaiá (Fonte: CEMIG) 
mínimas de 4,28 $\left(\mathrm{t} \mathrm{d}^{-1}\right)$ nos meses de junho a agosto. Referida variação está correlacionada com a variabilidade da descarga líquida com uma relação linear sem efeito de histereses; em razão da dificuldade de acesso à série completa, os estudos ficaram limitados a um período de 4 anos (1999 a 2002).

De acordo com Braga et al. (2012) o conhecimento do comportamento hidrossedimentológico em uma bacia hidrográfica é fundamental para o planejamento e desenvolvimento sustentáveis; no reservatório de Três Marias a aplicação desse conhecimento viabiliza o planejamento e a geração de eletricidade através de hidrelétricas uma vez que a vida útil das usinas é determinada com base na retenção e na distribuição dos sólidos em seu reservatório.

$\mathrm{Na}$ série temporal de 12 anos as imagens MODIS, composta de 8 dias e processada com o algoritmo MOD3R foram classificadas e selecionadas por critérios de qualidade, como ângulo da incidência solar, ângulo de visada do sensor, presença de aerossois e presença ou ausência de nuvens (banda de qualidade MODIS A1), mas nem todas as imagens foram aproveitadas para estimar endmember de água. Das 1024 imagens analisadas (Terra e Aqua), 82\% obtiveram qualidade aceitável e foram usadas para se obter estimativas de reflectância da água; os $18 \%$ restantes das imagens não foram aproveitados, o que pode estar relacionado à resolução efetiva do sensor, cobertura de nuvens (período chuvoso), largura do braço (poucos pixels puros amostrados) e da hidrologia (variação do nível do reservatório).

Os resultados apresentados na Figura 5 são séries temporais de reflectância da água processada com o algoritmo MOD3R ao longo do braço do Rio Indaiá para as 8 regiões de interesse (Figura 3); as análises foram centradas na banda 2 das imagens MODIS (produto de reflectância da superfície $250 \mathrm{~m}$ ) referentes ao comprimento de onda do infravermelho (NIR).

Os gráficos obtidos através das análises da reflectância da água permitem observar: 1) um ciclo sazonal bem definido (altos valores de reflectância nos meses de chuva e baixos valores nos meses de seca); 2) uma forte variação da reflectância ao longo do braço do reservatório no sentido da montante à jusante com valores de reflectância que chegam a 2000 (máscara 1) e baixos valores de 100 e 50 (máscara 7 e 8); 3) altos valores de reflectância nos anos de 2000 a 2004 das primeiras máscaras analisadas; esta diferença na reflectância pode estar relacionada diretamente com o nível da água do reservatório Três Marias, que nos anos de 2000 a 2003 operou com o nível entre 10 a 30\% de sua capacidade nos meses mais secos (ONS, 2012).

A possibilidade de relacionar a concentração de sedimentos em águas continentais usando as respostas espectrais das imagens de sensoriamento remoto, foi investigada por vários estudos incluindo Novo et al. (1989); Doxaran et al. (2002; 2005) e Martinez et al. (2009). Esses trabalhos relatam forte correlação entre sedimentos em suspensão e a resposta espectral das imagens, porém esta relação depende do comprimento de onda. A maioria dos estudos concorda que a melhor correlação entre sedimentos em suspensão e reflectância está centrada entre os 600 e $900 \mathrm{~nm}$ em águas continentais.
A Figura 6 apresenta a relação da série de descarga sólida diária da estação de monitoramento hidrossedimentológico do Rio Indaiá (Figura 4), com as estimativas de reflectância da água dos dados MODIS (8 dias) nas bandas do vermelho e infravermelho em 2 ciclos sazonais hidrológicos para as máscaras definidas na Figura 3.

Os dados de reflectância MODIS da máscara 1 não se relacionam muito bem com os dados de vazão sólida no período de seca no ano de 2001. É provável que o baixo nível da água no reservatório naquele período tenha favorecido o crescimento de vegetação ciliar ou plantas aquáticas no início do braço do rio. Este tipo de alvos pode ter gerado os altos valores de reflectância na máscara 1 no ano de 2001, haja vista que a assinatura está próxima ao padrão da resposta espectral de alvos como vegetação (baixo no vermelho e alto no infravermelho) e não com a reflectância da água.

Seguindo com as análises das outras máscaras é possível notar uma boa relação entre a resposta da reflectância dos dados MODIS com a descarga sólida de sedimentos. Quando a descarga sólida aumenta no rio a reflectância no braço calculada nas bandas do vermelho e infravermelho, aumenta. Observa-se que esta relação é contínua em todos os ciclos sazonais; outro padrão de variação que pode ser visto nos dados MODIS é a diminuição da reflectância no sentido montante-jusante à medida que as máscaras se afastam do encontro do rio com o reservatório.

Para uma visualização melhor da relação entre reflectância dos dados MODIS e a descarga sólida de sedimentos, a Figura 7 mostra, com mais detalhes, a resposta das bandas vermelha e infravermelha na máscara 2 para os meses junho-2000/ junho-2001 e junho-2001/junho-2002. Conclui-se que alguns dados de reflectância do MODIS não foram aproveitados devido à baixa resolução espacial do sensor e à quantidade de nuvens existentes no período de chuva problemas que, sem dúvida, limitam o algoritmo MOD3R em encontrar pixels puros de água no braço do reservatório e, assim, reduzem significativamente a quantidade de dados de reflectância neste período.

A resposta do comportamento temporal da reflectância ao longo do braço do Rio Indaiá possibilitou entender alguns fatores que controlam o comportamento da entrada do fluxo de sedimentos. A Figura 8 apresenta médias mensais para cada máscara analisada. As médias foram obtidas através do processamento da série temporal de imagens MODIS (Terra e Aqua) usando-se o algoritmo MOD3R. Os dados de ambos os satélites foram fusionados e filtrados usando-se a banda de qualidade do produto MODIS A1; em seguida, foram obtidas médias de cada máscara para todos os meses do ano.

A análise das médias de reflectância da superfície da água permitiu identificar: 1) a existência de fortes valores de reflectância nos meses de chuva, principalmente nas 2 primeiras máscaras; 2 ) as máscaras 1 e 2 obtiveram valores de reflectância maiores que as outras regiões (descarga contínua de sedimentos) ao longo de toda a série temporal; 3) o gradiente espacial de reflectância é maior nos meses de chuva (janeiro, fevereiro, 

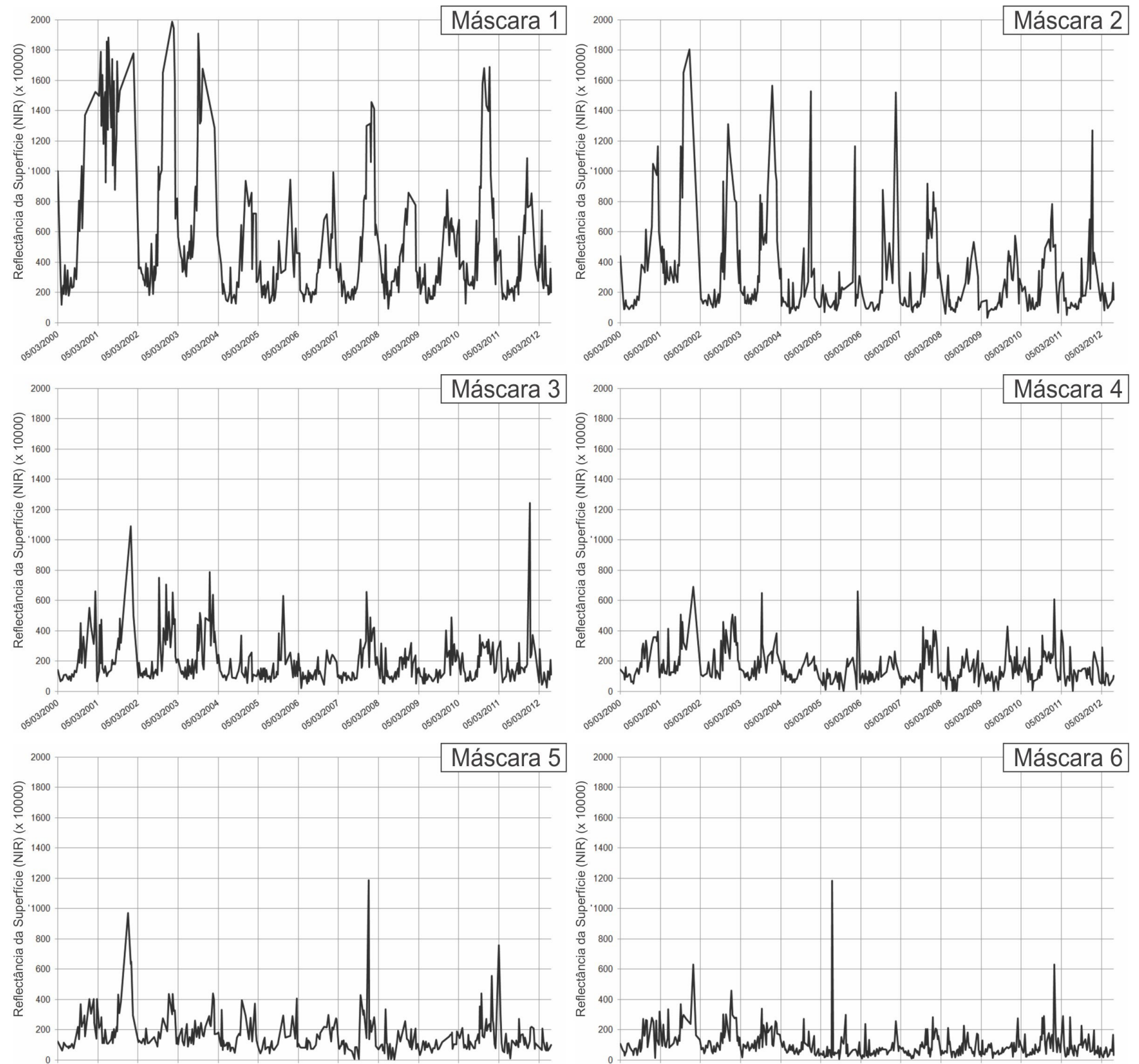

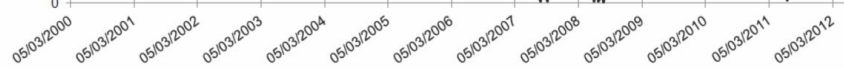
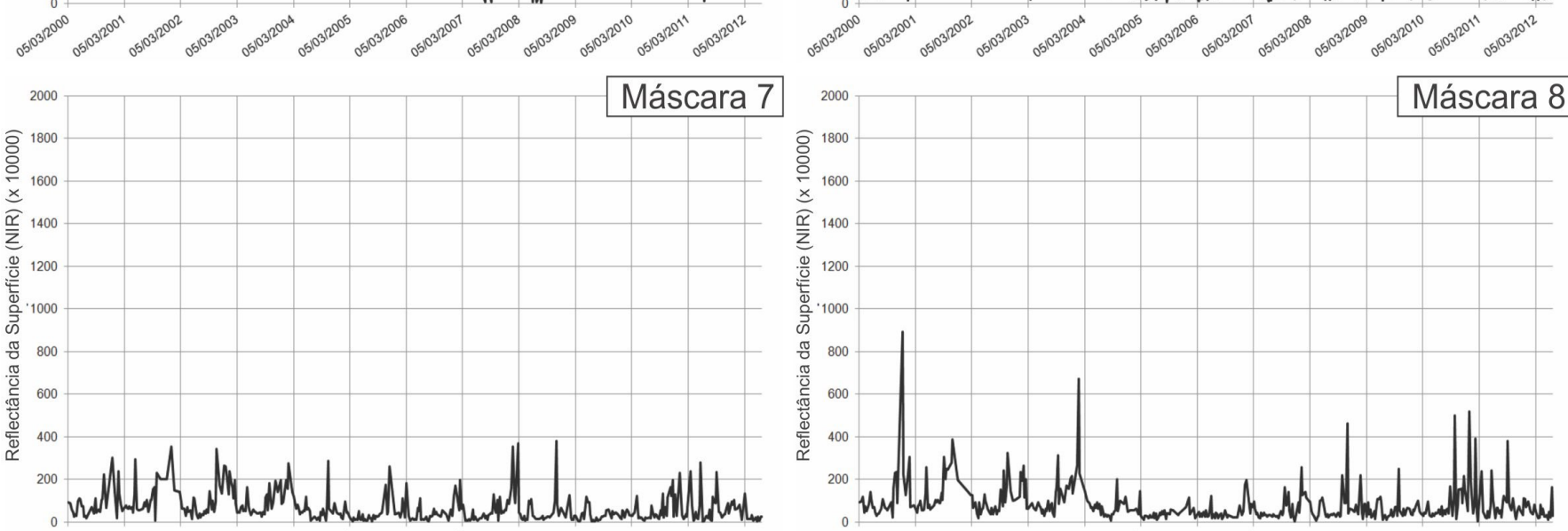

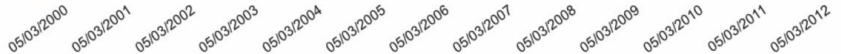

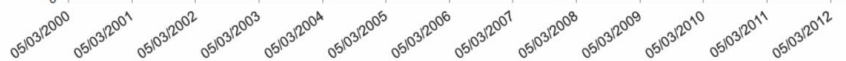

Figura 5. Estimativas de reflectância da superfície da água na região do infravermelho do MODIS nas máscaras analisadas no braço do Rio Indaiá, reservatório Três Marias, MG 

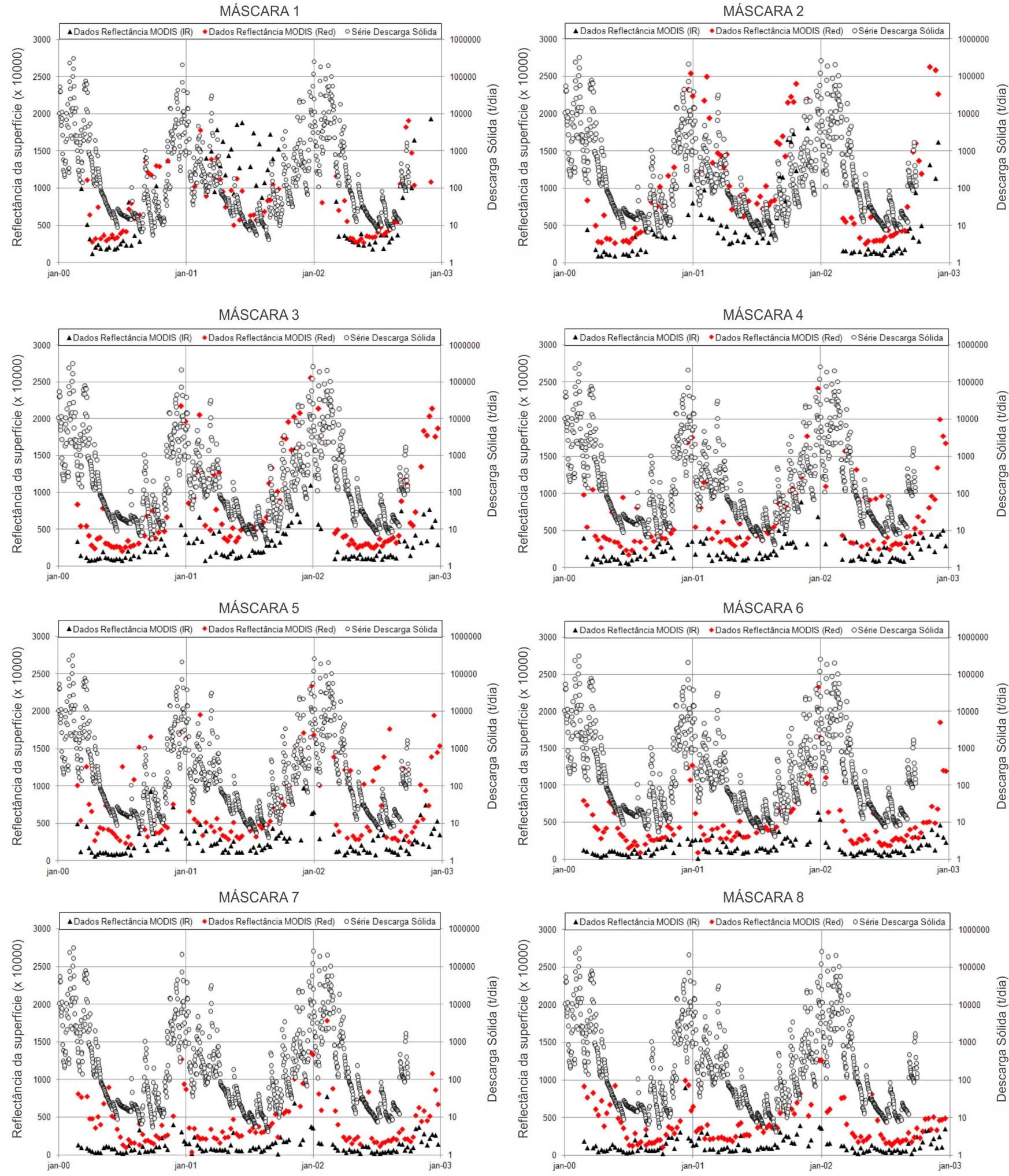

Figura 6. Fluxo da vazão sólida diária na estação do Rio Indaiá em função da reflectância da superfície das imagens MODIS (8 dias), nos comprimentos de onda do vermelho e infravermelho

março, outubro, novembro e dezembro; 4) na máscara 5, 6, 7 e 8 apresentaram um aumento dos valores médios de reflectância no mês de maio. Este último fato pode ser associado ao uso agrícola intenso das margens do reservatório a partir da máscara 5; as margens do braço parecem estar protegidas pela vegetação nativa nas imagens do Google Earth; é possível que o aumento da reflectância no mês de maio seja resultante das práticas de cultivo neste período (safra).

As estimativas de reflectância foram calculadas para cada mês do ano, permitindo um estudo espaço-temporal do processo de fluxo de sedimentos ao longo de 12 anos. A Figura 9 mostra médias de reflectância para cada mês do ano em função 
A.

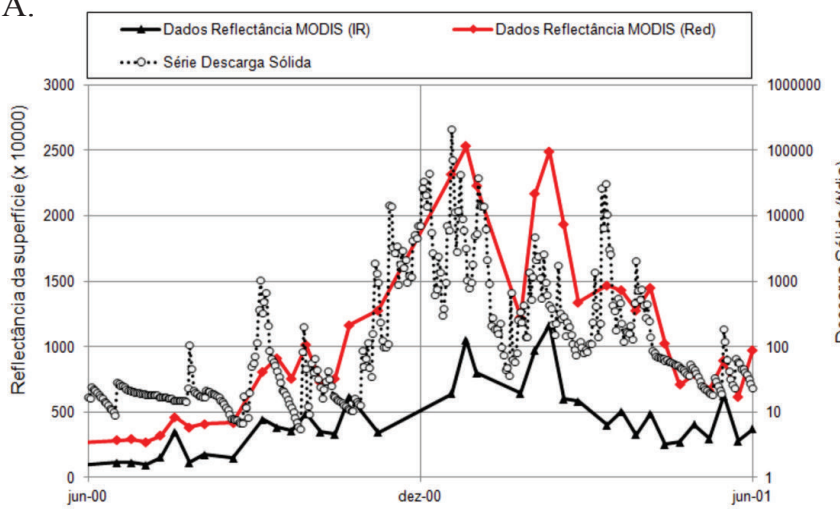

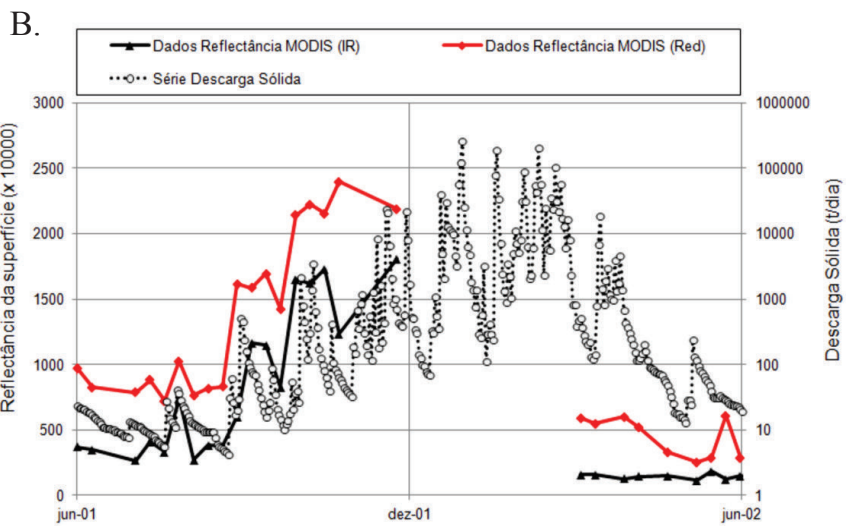

Figura 7. Fluxo da vazão sólida diária na estação do Rio Indaiá em função da reflectância da superfície das imagens MODIS (8 dias), nos comprimentos de onda do vermelho e infravermelho. Detalhe da máscara 2 para os meses junho-2000/junho-2001 (A) e junho-2001/junho 2002 (B)

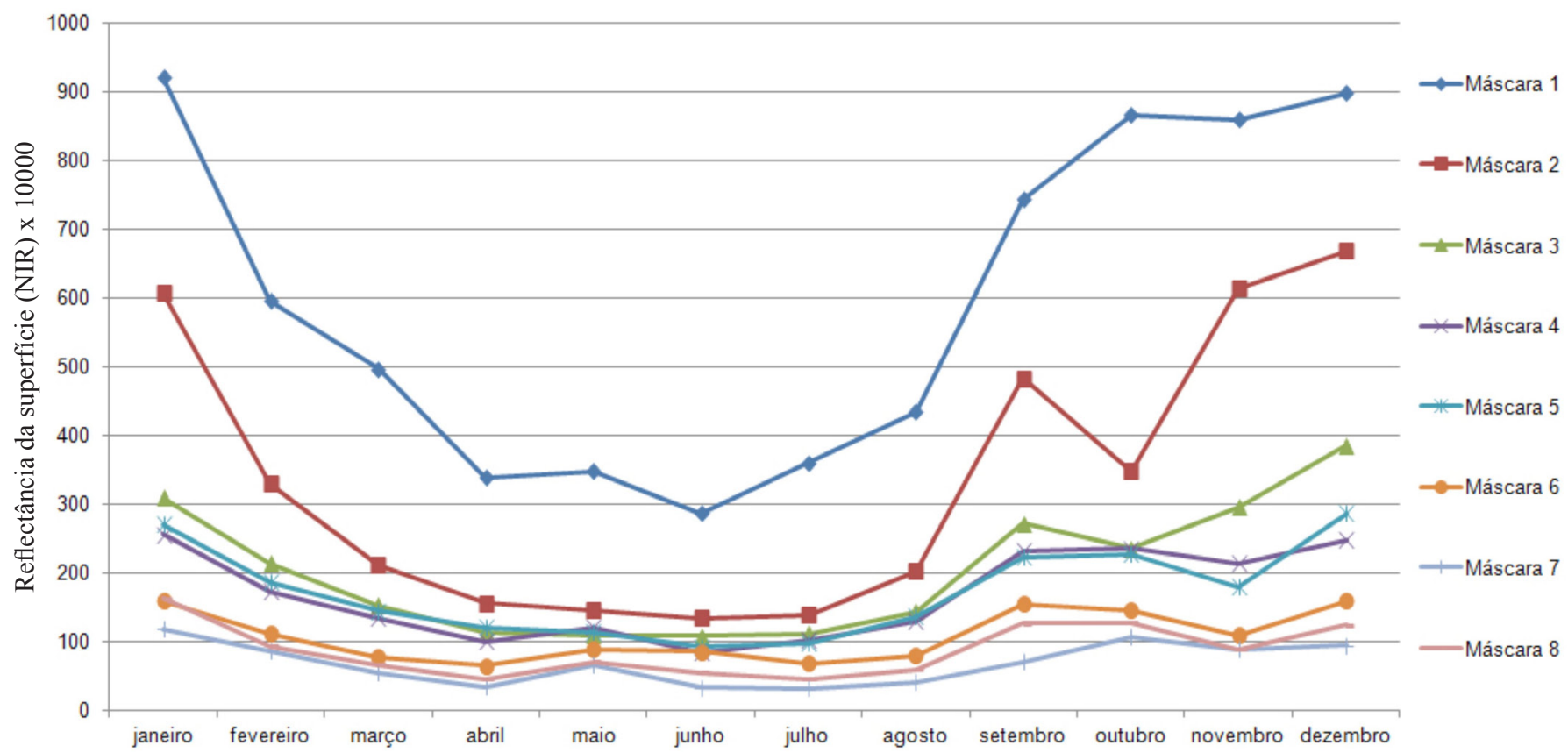

Figura 8. Médias mensais estimada por satélite (período 2000-2012) da reflectância da superfície das máscaras analisadas no braço do Rio Indaiá, reservatório Três Marias, MG

da distância das 8 máscaras analisadas. Observou-se que: 1) os valores de reflectância diminuem em função da distância das regiões analisadas no sentido montante-jusante; 2) altos valores de reflectância nos meses de chuvas, principalmente nos primeiros $5 \mathrm{~km}$; 3) na máscara 5 ocorreu um aumento nos valores médios da reflectância. No braço do reservatório existem pequenos afluentes que podem influenciar diretamente na entrada de sedimentos alterando a cor da água em diferentes pontos.

Carvalho (2008) explica que os sedimentos carregados pelo rio para dentro de um reservatório não são distribuídos uniformemente, as partículas maiores, como pedregulhos e areias grossas são as primeiras a se depositar, enquanto os sedimentos mais finos adentram no reservatório. Embora os produtos de reflectância da superfície do sensor MODIS tenham resolução espacial média $(250 \mathrm{~m})$ nas bandas do vermelho e infravermelho, suas imagens compostas de 8 dias permitem uma avaliação temporal melhor e podem identificar mudanças na concentração e deposição de sedimentos em reservatórios. Referidas informações podem auxiliar na identificação das variações do fluxo de sedimentos e, em consequência, da qualidade da água.

Em virtude da resolução espacial do sensor MODIS, a extração da reflectância da água usando as imagens de satélite poderia ser comprometida pela largura do braço, especialmente nos períodos de seca quando o nível do reservatório baixa. Nos períodos de seca extremas entre 2000 e 2002, uma assinatura espectral de vegetação foi detectada porém apenas nas regiões mais próximas à entrada do Rio Indaiá; nota-se, com exceção deste período, que as imagens de satélite permitem monitorar, de maneira quase continua, a qualidade das águas no braço do Rio Indaiá.

Os resultados apresentados da série temporal de reflectância dos dados MODIS mostraram boa relação com os dados de vazão sólida coletados dentro do Rio Indaiá; desta forma, comprovou-se que as imagens de satélite poderiam 


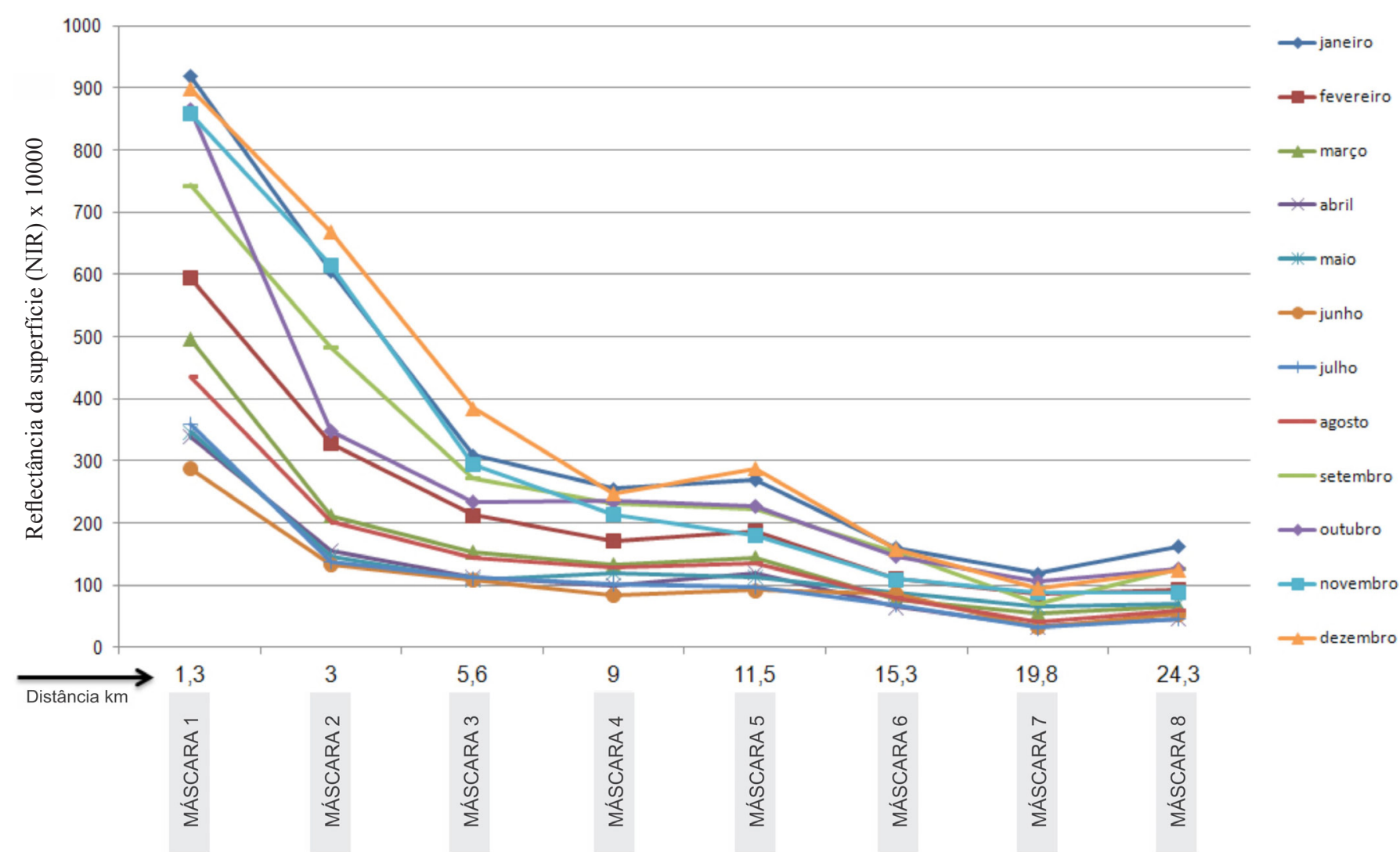

Figura 9. Médias mensais estimadas por satélite (período 2000-2012) da reflectância da superfície em todos os meses do ano em função da distância ao longo do braço do Rio Indaiá, reservatório Três Marias, MG

servir como solução alternativa de baixo custo em relação aos métodos tradicionais usados pela CEMIG, para entender os processos de assoreamento dentro do reservatório e serem integrados ao sistema de monitoramento do reservatório de Três Marias.

\section{Conclusões}

1. Os dados de reflectância da superfície do sensor MODIS estão relacionados, de maneira robusta, aos dados de concentração de sedimentos em todos os ciclos hidrológicos da região.

2. O algoritmo MOD3R mostrou-se eficiente em estimar valores de reflectância no braço do reservatório mas teve dificuldades em estimar pixels puros em algumas regiões devido à largura do braço e à variação do nível do reservatório.

3. O percentual de registros válidos estimados nas imagens MODIS (> 80\%) atendem aos requisitos para um monitoramento operacional do fluxo de sedimentos; em particular, o número de observações obtidas pelos satélites pode auxiliar no monitoramento de todo o ciclo hidrossedimentológico da região.

4. Os dados de reflectância das imagens MODIS podem ser usados como solução alternativa para monitorar alguns parâmetros de qualidade de água, como a concentração de sedimentos em braços de reservatórios permitindo, deste modo, uma análise temporal que extrapola a série histórica de descarga sólida disponível.

\section{Agradecimentos}

Os autores agradecem ao Programa de Pós-Graduação em Geociências Aplicadas do Instituto de Geociências (IG) da Universidade de Brasília; à FINEP, que financiou o projeto "Avaliação e Gestão do Assoreamento de Reservatórios" (convênio 04.10.0071.00); ao CNPq, pela disponibilização das bolsas de pesquisa vinculada ao projeto da FINEP para os dois primeiros autores; ao ICMBio, pelo apoio e logística da coleta de dados em campo; à CEMIG, pelo apoio e disponibilização dos dados hidrossedimentométricos da estação do Rio Indaiá e ao IRD, pela disponibilização do aplicativo MOD3R; à ESRI, pela disponibilização do pacote de ferramentas que compõem a família ArcGis 10, por intermédio do contrato № 2011 MLK 8733 e à IMAGEM, pelo apoio e viabilidade da concretização do termo de uso entre o IG e à ESRI, pelo suporte aos softwares.

\section{Literatura Citada}

ANEEL - Agência Nacional de Energia Elétrica. Sistema de informações georreferenciadas de energia e hidrologia. Brasília: ANEEL, 2000. CD-Rom

Bradl, H. B. Adsorption of heavy metal ions on soils and soils constituents, Journal of Colloid and Interface Science, v.277, p.1-18. 2004.

Braga, C. C.; Rocha, I. R.; Cabral, J. B. P.; Barcelos, A. A. Análise de descarga sólida em suspensão dos afluentes do reservatório da UHE Barra dos Coqueiros-GO. Revista Geonorte, v.3, p.495-505. 2012. 
Carvalho, N. O. Hidrossedimentometria prática. 2.ed. Rio de Janeiro: Editora Interciências Ltda. 2008. 599p.

Chanson, H. Extreme reservoir sedimentation in Australia: A review. International Journal of Sediment Research, v.13, p.55-63, 1998.

Doxaran, D.; Cherukuru, R.; Lavender, S. Use of reflectance band ratios to estimate suspended and dissolved matter concentrations in estuarine waters. International Journal of Remote Sensing, v.26, p.1763-1770. 2005.

Doxaran, D.; Froidefond, J. M.; Lavender, S.; Castaing, P. Spectral signature of highly turbid waters. Application with SPOT data to quantify suspended particulate matter concentrations. Remote Sensing of Environment, v.81, p.149-161, 2002.

Duan, H. T.; Zhang, Y. Z.; Zhan, B.; Song, K. S.; Wang, Z. M. Assessment of chlorophyll - A concentration and trophic state for Lake Chagan using Landsat TM and field spectral data. Environmental Monitoring and Assessment, v.129, p.295-308, 2007.

Ferreira, A. B.; Pereira Filho, W. Avaliação da reflectância espectral de corpos d'água em Santa Maria-RS, por meio de espectrorradiometria de campo. Revista Geoambiente, v.13, p.194-207, 2009.

GEMS - Global Environment Monitoring System. Improving global water quality monitoring. Washington: United Nation Environment Program - 2003. 18p. Technical Advisory Paper n.1.

Hooper, R. P. Sediment sampling in the national stream quality accounting network. In: U. S. Geological Survey. Sediment Workshop, Atlanta, 1997. Proceedings... Atlanta: U.S. Geological Survey, 1997. CD Rom

Kilham, N. E.; Roberts, D. Amazon River time series of surface sediment concentration from MODIS, International Journal of Remote Sensing, v.32, p.2659-2679. 2011.

Kirk, J. T. O. A Monte Carlo study of the nature of the underwater light field in, and the relationships between optical properties of, turbid yellow waters. Australian Journal of Marine \& Freshwater Research, v.32, p.517-532. 1981.

Liu, Y.; Islam M. A.; Gao, J. Quantification of shallow water quality parameters by means of remote sensing. Progress in Physical Geographyv, v.27, p.24-43, 2003.

Macedo. I. L. Estudos de modelos em ambiente de geoprocessamento para previsão de erosão e assoreamento de reservatórios: O caso da Bacia do Rio Indaiá - UHE Três Marias, MG. Brasília: DECA/UnB, 2009. 340p. Tese Doutorado

Mannino A.; Russ M. E.; Hooker S. B. Algorithm development and validation for satellite-derived distributions of DOC and CDOM in the U.S. Middle Atlantic Bight. Journal of Geophysical Research, v.113, p.19-42, 2008.
Martinez, J. M.; Guyot, J. L., Filizola, N., Sondag, F. Increase in suspended sediment discharge of the Amazon River assessed by monitoring network and satellite data. Catena, v.79. p.257-264. 2009.

Mertes, L. A. K., Smith, C. T., Adams, J. B. Estimating suspended sediment concentrations in surface waters of the Amazon River wetlands from Landsat images: Remote Sensing of Environment, v.43, p.281-301. 1993.

Nascimento, R. F. F; Alcântara, E.; Kampel, M.; Stech, J. L. Caracterização limnológica do reservatório hidrelétrico de Itumbiara. Ambi-Agua, v.6, p.143-156, 2011.

Novo, E. M. L. M.; Hamsom, J. D.; Curran, P. J. The effect of sediment type on the relationship between reflectance and suspended sediment concentrations: International Journal of Remote Sensing, v.10, p.1283-1289, 1989.

Onderka, M.; Rodný, M. Can suspended sediment concentration be estimated from multispectral imagery using only imagederived information? Journal of the Indian Society of Remote Sensing, v.38, p.85-97. 2010.

ONS - Operador Nacional do Sistema Elétrico. Volume útil dos principais reservatórios. <http://www.ons.org.br/historico/ percentual_volume_util.aspx>. 10 Nov. 2012.

Rudorff, C. M.; Novo, E. M. L. M.; Galvão, L. S.; Pereira Filho, W. Análise derivativa de dados hiperespectrais medidos em nível de campo e orbital para caracterizar a composição de águas opticamente complexas na Amazônia. Acta Amazonica, v.37, p.269-280. 2007.

Simis S. G. H.; Peters S W. M.; Gons H J. Remote sensing of the cyanobacterial pigment phycocyanin in turbid inland water. Limnology and Oceanography, v.50, p.237-245. 2005.

Tonello, K. C. Análise hidroambiental da bacia hidrográfica da cachoeira das pombas, guanhães, MG. Viçosa: UFV, 2005. 85p. Dissertação Mestrado

Valério, A. M.; Kampel, M.; Stech, J. L. Correlação de dados de reflectância de sensoriamento remoto estimados com dados do sensor MODIS e medidas in situ. In: Simpósio Brasileiro de Recursos Hídricos, 15. Curitiba. Anais... Curitiba: ABRH, 2011. p.5100-5107.

Villar, R. E.; Martinez, J. M.; Guyot, J. L.; Fraizy, P.; Armijos, E.; Crave, A.; Bazán, H.; Vauchel, P.; Lavado, W. The integration of field measurements and satellite observations to determine river solid loads in poorly monitored basins. Journal of Hydrology, v.44, p.45-54. 2012.

Wang, J. J.; Lu, X. X. Estimation of suspended sediment concentrations using Terra MODIS: An example from the Lower Yangtze River, China. Science of the Total Environment, v.408, p.1131-1138, 2010.

Zhu, W.; Yu, Q.; Tian, Y. Q.; Chen, R. F.; Gardner, G. B. Estimation of chromophoric dissolved organic matter in the Mississippi and Atchafalaya river plume regions using above-surface hyperspectral remote sensing. Journal of Geophysical Research, v.116, p.1-22, 2011. 